\title{
Calcium-siRNA Nanocomplexes Optimized by Bovine Serum Albumin Coating Can Achieve Convenient and Efficient siRNA Delivery for Periodontitis Therapy
}

This article was published in the following Dove Press journal:

International Journal of Nanomedicine

\section{Yang Wang ${ }^{\prime}$ \\ Wen Song ${ }^{2}$ \\ Yi Cui ${ }^{3}$ \\ Yang Zhang' \\ Shenglin $\mathrm{Mei}^{4}$ \\ Qintao Wang'}

'State Key Laboratory of Military Stomatology, Department of Periodontology, School of Stomatology, Air Force Medical University, Xi'an, People's Republic of China; ${ }^{2}$ State Key Laboratory of Military Stomatology, Department of Prosthetic Dentistry, School of Stomatology, Air Force Medical University, Xi'an, People's Republic of China; ${ }^{3}$ Equipment Department, Xijing Hospital, Air Force Medical University, Xi'an, People's Republic of China; ${ }^{4}$ Department of Prosthodontics, College of Stomatology, Xi'an Jiaotong University, Xi'an, People's Republic of China
Correspondence: Qintao Wang

Department of Periodontology, School of Stomatology, Air Force Medical

University, No. 145 West Changle Road, Xi'an 710032, People's Republic of China

Tel +862984776096

Fax +00862983223047

Email yznmbk@fmmu.edu.cn
Purpose: Reducing toxicity, immunogenicity, and costs of small interfering RNAs (siRNA) carrier materials are key goals for RNA interference (RNAi) technology transition from bench to bed. Recently, calcium ions $\left(\mathrm{Ca}^{2+}\right)$ have garnered attention as a novel, alternative material for delivering siRNA to cells. However, the tolerance for $\mathrm{Ca}^{2+}$ concentration varies in different cell types, which has limited its applications in vivo. Bovine serum albumin (BSA) can bind to $\mathrm{Ca}^{2+}$ through chelation. Moreover, BSA is a favorable coating material for nanoparticles owing to its excellent biocompatibility. Therefore, we hypothesized that coating $\mathrm{Ca}^{2+}$-siRNA with BSA helps buffer $\mathrm{Ca}^{2+}$ toxicity in vivo.

Methods: BSA-Ca ${ }^{2+}$-siRNA nanoparticles were prepared, and the size, shape, encapsulation, and release efficiency were characterized using atomic force microscopy, scanning electronic microcopy, and gel electrophoresis. Binding nanoparticles were evaluated using attenuated total reflection-Fourier-transform infrared spectroscopy. The cellular uptake, intracellular release, cytotoxicity, and gene knockdown of nanoparticles were evaluated in periodontal ligament stem cells (PDLSCs) using laser-scanning confocal microscope, flow cytometry, and real-time quantitative polymerase chain reaction.

Results: BSA and $\mathrm{Ca}^{2+}$-siRNA could form a stable nano-scale complex $(\sim 140 \mathrm{~nm}$ in diameter). The nanocomplexes could maintain siRNA release for more than 1 week in neutral phosphate-buffered saline (PBS) and could induce accelerated degradation in acidic PBS ( $\mathrm{pH}$ 5.0). The nanoparticles were taken up by the cells, primarily through macropinocytosis, and were then released intracellularly through the acidification of endosomes/lysosomes. Importantly, the $\mathrm{BSA}_{-} \mathrm{Ca}^{2+}$ carrier had high transfection efficiency and biocompatibility both in vitro and in vivo. To demonstrate the therapeutic potential of our BSA coating-optimized $\mathrm{Ca}^{2+}$-siRNA technology, we showed that BSA-Ca ${ }^{2+}$-siWWP1 complexes strongly enhanced the osteogenic differentiation of inflammatory PDLSCs.

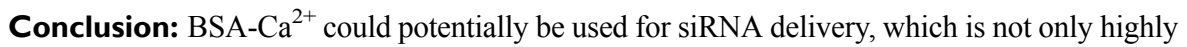
efficient and cost-effective but also biocompatible to host tissues owing to the BSA coating.

Keywords: bovine serum albumin, calcium ions, osteogenic differentiation, small interfering RNA

\section{Introduction}

Owing to its high specificity and effectiveness, RNA interference (RNAi) is used in biomedicine to study target genes, and the potential therapeutic applications of this technique are emerging. ${ }^{1}$ However, the clinical implementation of RNAi therapy is 
hampered by factors such as enzymatic degradation, cell targeting, uptake, and endosomal escape under physiological environments. ${ }^{2}$ To overcome these limitations, research has focused on the development of improved small interfering RNAs (siRNA) carrier materials. ${ }^{3}$ Most carrier materials are cationic lipids or polymers synthesized by exogenous chemical methods and are commonly toxic and immunogenic. ${ }^{4-6}$ Moreover, the preparation of such nanocarriers often requires complex technology and is associated with a high cost. ${ }^{7}$ Therefore, developing convenient and biocompatible siRNA vectors remains a challenge. Reportedly, calcium ions $\left(\mathrm{Ca}^{2+}\right)$ are adsorbed on siRNA surface and spontaneously form uniform-sized $\mathrm{Ca}^{2+}$-siRNA nanoparticles with an average diameter of $100 \mathrm{~nm}^{2,5,7}$ These nanoparticles can be taken up by mesenchymal stem cells (MSCs) or some cancer cells, resulting in efficient, convenient, and low-cost siRNA delivery. ${ }^{2,7}$ However, high $\mathrm{Ca}^{2+}$ levels are toxic in vivo and can lead to blood coagulation and acute inflammation. ${ }^{8,9}$ In addition, we found that in vitro transfection using medium containing high $\mathrm{Ca}^{2+}$ concentrations and without fetal bovine serum (FBS) can induce cell death. Therefore, we hypothesized that FBS, particularly the protein contained within it, plays a crucial role in alleviating $\mathrm{Ca}^{2+}$ toxicity.

Bovine serum albumin (BSA) is the primary component of FBS and can be combined with a variety of cations, anions, and other small molecule substances..$^{10}$ BSA addition to in vitro cell cultures without FBS can confer physiological and mechanical protection. ${ }^{11}$ Furthermore, BSA can also be used as a carrier for various drug-delivery applications due to its favorable properties such as high biodegradability, low toxicity, low antigenicity, high stability, ability for long-term storage, and ease of production. ${ }^{12,13}$ In addition, studies have demonstrated that $\mathrm{Ca}^{2+}$ can chelate with BSA to form microspheres. ${ }^{14}$ Therefore, we hypothesized that coating $\mathrm{Ca}^{2+}$-siRNA with BSA can help buffer $\mathrm{Ca}^{2+}$ toxicity and can be used for in vivo applications. Herein, we fabricated BSA-Ca ${ }^{2+}$-siRNA nanoparticles, to the best of our knowledge, for the first time and presented a scheme of the fabrication flow: premixing $\mathrm{Ca}^{2+}$ and siRNA to form nanocomplexes prior to BSA coating on the surface of the $\mathrm{Ca} / \mathrm{siRNA}$ complexes. The cellular uptake, release, and transfection of nanoparticles were evaluated. Transfection and knockout efficiency were optimized by adjusting the BSA or $\mathrm{Ca}^{2+}$ concentrations and the therapeutic potential of the BSA-Ca ${ }^{2+}$ delivery system was evaluated through cytotoxicity and hemolysis experiments.
To demonstrate the therapeutic potential of this BSA$\mathrm{Ca}^{2+}$-siRNA transfection technology, we observed the effect of BSA-Ca ${ }^{2+}$-siWWP1 on the promotion of osteogenesis in inflammatory PDLSCs. At present, available treatment options for periodontitis mostly focus on controlling inflammation; however, it remains difficult to restore the absorbed alveolar bone to normal. Therefore, the difficulty in delaying bone resorption is also a key limitation in the clinical treatment of periodontitis. The WW domain containing E3 ubiquitin protein ligase 1 (WWP1) is a member of the C2-WW-HECT family of E3 ligases. ${ }^{15,16}$ Chronic exposure to tumor necrosis factor- $\alpha$ (TNF- $\alpha)$ reduces the osteogenic potential of MSCs by increasing WWP1-mediated degradation of Runx 2 and JunB proteins. ${ }^{17,18}$ Targeted WWP1 knockdown can significantly improve bone phenotype in vivo and in vitro through signaling pathways related to bone morphogenetic proteins. ${ }^{17,19}$ Therefore, we delivered WWP1 siRNA via a BSA-Ca ${ }^{2+}$ delivery platform for inducing osteogenesis in inflammatory periodontal ligament stem cells (PDLSCs).

\section{Materials and Methods Preparation of BSA-Ca ${ }^{2+}$-siRNA Complexes}

The sequences of gene-targeting siRNAs (Sangon, China) dissolved in RNase-free water are shown in Table S1. HEPES (10 mM, pH 7.4), BSA $(5-5,000 \mu \mathrm{g} / \mathrm{mL})$, siRNA $(20 \mu \mathrm{M})$, and $\mathrm{CaCl}_{2}(0.5-2 \mathrm{M}$ stock concentrations) solutions were freshly prepared in RNase-free water at a ratio of 1:1:1:1 to obtain the molar ratios. The scheme of the manufacturing process is as follows. First, equal volumes of $\mathrm{CaCl}_{2}$ and siRNA were vortexed for $45 \mathrm{~s}$ and incubated for $25 \mathrm{~min}$ at $4^{\circ} \mathrm{C}$. $^{7}$ Then, an equal volume of BSA was added, and the mixture was vortexed for $45 \mathrm{~s}$, followed by incubation for $25 \mathrm{~min}$ at $4^{\circ} \mathrm{C}$. Finally, an equal volume of HEPES was added, and the mixture was vortexed for $45 \mathrm{~s}$, followed by incubation for $25 \mathrm{~min}$ at $4{ }^{\circ} \mathrm{C}$ prior to use. Lipofectamine 2000 (Invitrogen, USA) transfection complex was prepared in Opti-Minimum Essential Medium (Opti-MEM; Gibco, USA) according to the manufacturer's instructions. Other reagents, unless specified otherwise, were obtained from Sigma-Aldrich (USA).

\section{Cell Culture}

PDLSCs were derived from fresh premolars without caries that were removed for orthodontic purposes from healthy individuals aged 19-31 years. All study donors provided 
informed consent, and all procedures followed the guidelines of the Ethics Committee of the Stomatological Hospital of Air Force Medical University (approval Number IRB-REV-2017012). PDLSCs were cultured using limiting dilution analysis, and third-to-fifth generation polyclonal PDLSCs were selected for use in this study. According to the manufacturer's instructions, a lentiviral vector expressing green fluorescent protein (GFP: pRLenti-EF1a-EGFP-P2A-Puro-CMV-MCS-3Flag; Obio, China) was used to infect PDLSCs to obtain GFPPDLSCs. PDLSCs, with and without GFP expression, were cultured in $\alpha$-Minimum Essential Medium $(\alpha-$ MEM; Hyclone, USA), supplemented with L-glutamine (Lonza, USA) and 10\% FBS (Gibco, USA). Under conventional transfection conditions, PDLSCs were seeded in a 24-well plate at a density of 22,000 cells/well. After 24 $\mathrm{h}$, PDLSCs were incubated with transfection complexes for $3.5 \mathrm{~h}$ and replaced with fresh medium.

\section{Characterization of Complexes}

Three microliters of $\mathrm{BSA}^{-\mathrm{Ca}^{2+}}{ }^{2}$-siRNA complexes were deposited on the surface of the silicon wafer. After $20 \mathrm{~min}$, the surface was dried under $\mathrm{N}_{2}$ gas. The morphology of samples was observed via the tapping mode of atomic force microscopy (AFM; Agilent, USA). Apparent diameters of the particles were analyzed using the SPM software (Agilent, USA). The samples were characterized using scanning electronic microcopy (SEM; Thermo Fisher, USA) in a low-dose imaging mode of $5 \mathrm{kV}$. The particle size was measured using the ImageJ software (National Institutes of Health, USA). Zeta potential and dynamic light scattering (DLS)-measured particle size distribution of complexes were obtained using a Zetasizer Nano (Malvern Panalytical, UK). Approximately $100 \mu \mathrm{L}$ samples were placed in centrifuge tubes, dried in a lyophilizer (Biocool, China) at $-80^{\circ} \mathrm{C}$ for $24 \mathrm{~h}$, and chemically characterized using attenuated total reflection-Fouriertransform infrared spectroscopy (FTIR; Nexus-670, USA).

\section{Cargo Loading and Release}

The loading efficiency of the siRNAs was evaluated by gel electrophoresis. siRNA complexes were loaded onto a gel consisting of $3.5 \%$ agarose and SYBR Gold, and then electrophoresed in TAE buffer solution at $110 \mathrm{~V}$ and $40 \mathrm{~mA}$. The obtained siRNA signals were imaged using a Gel Doc 2000 (Bio-Rad, USA). BSA-Ca ${ }^{2+}$-siRNA particles were suspended and incubated in neutral (pH 7.4) or acidic (pH 5.0) PBS at $37^{\circ} \mathrm{C}$ for $24 \mathrm{~h}$. Subsequently, a spectrophotometer (Thermo Fisher, USA) was used to analyze the siRNA content of the supernatant of the centrifuged samples. The centrifuged particles were re-immersed in PBS and allowed to continue degradation. This step was repeated for 7 days. ${ }^{20}$

\section{Uptake and Release Pathways}

PDLSCs (P3) were seeded on glass-bottomed dishes (MatTek, USA) at a density of 42,000 cells/well. After 24 h, PDLSCs were washed with PBS and treated with each of the inhibitors at its selected concentration (Table 1) for 1 h. ${ }^{5,21}$ Then, BSA-Ca ${ }^{2+}$-siRNA nanocomposites $(5 \mu \mathrm{M}$ siRNA, $125 \mu \mathrm{g} / \mathrm{mL} \mathrm{BSA}$, and $250 \mathrm{mM} \mathrm{CaCl}_{2}$ ) that were freshly diluted 1:50 in $\alpha$-MEM were added, and cells were incubated for a further $3.5 \mathrm{~h}$. Finally, PDLSCs were analyzed using a flow cytometer (Beckman Coulter, USA) and fixed with $4 \%$ paraformaldehyde. The cytoskeleton was stained with AF635-phalloidin (Thermo Fisher, USA), lysosomes/ endosomes were stained with LysoTracker (Invitrogen, USA), and the nuclei were stained with Hoechst (Invitrogen, USA). Images were obtained using a laserscanning confocal microscope (LSCM; Nikon, Japan).

\section{Knockdown Efficiency Quantification}

Knockdown efficiency was confirmed $72 \mathrm{~h}$ post-transfection. A fluorescence microscope (Olympus, Japan) was used to determine the fluorescence intensity of GFP-PDLSCs. Additionally, GFP-PDLSCs were collected by trypsinization and analyzed using a flow cytometer. The geometric mean of FL1 fluorescence intensity was calculated to evaluate the knockdown efficiency. The GFP silencing efficiency percentage was calculated using the following formulas:

$\%$ GFP $=$ MFI GFP in sample/MFI GFP in negative control $\times 100$, and

$\%$ Silencing $=100-\% \mathrm{GFP}$,

where MFI is the mean fluorescence intensity.

Table I Inhibitors: Functions and Concentrations

\begin{tabular}{|l|l|l|}
\hline $\begin{array}{l}\text { Inhibitor } \\
\text { Name }\end{array}$ & Pathway Inhibited & $\begin{array}{l}\text { Chosen } \\
\text { Concentration }\end{array}$ \\
\hline $\begin{array}{l}\text { Chlorpromazine } \\
\text { Genistein }\end{array}$ & Clathrin-dependent & $20 \mu \mathrm{M}$ \\
Amiloride & Actin-dependent & $50 \mu \mathrm{M}$ \\
Bafilomycin AI & V-ATPase inhibitor & $75 \mathrm{nM}$ \\
YM201636 & Maturation of early & $2 \mu \mathrm{M}$ \\
& endosome & \\
\hline
\end{tabular}

Abbreviation: V-ATPase, vacuolar-type $\mathrm{H}^{+}$ATPase. 


\section{Cytotoxicity Assay}

PDLSCs were seeded in 96-well plates (2,200 cells/well) and incubated in $\alpha$-MEM for $24 \mathrm{~h}$. Next, $\alpha$-MEM was replaced with serum-free medium containing BSA-Ca ${ }^{2+}$ siRNA complexes (dilution ratio with medium, 1:50). After $24 \mathrm{~h}$, PDLSCs were washed with PBS and incubated in $\alpha$-MEM for another $48 \mathrm{~h}$. Cell viability was determined using Cell Counting Kit-8 (CCK-8; Yeasen, China).

\section{Hemolysis Assay}

BALB/c mice ( 8 weeks old) were provided by the Air Force Medical University. All animal experiments were approved by the Experimental Animal Management Ethics Committee of Stomatological Hospital at the Air Force Medical University (approval number 2019-LAEI-kq-016) and were conducted in accordance with the Guidelines for Ethical Review of Laboratory Animal Welfare (GB/T 35892-2018) issued by National Technical Committee 281 on Laboratory Animal Science of Standardization Administration of China (SAC/TC 281).

Red blood cells (RBCs) were obtained by centrifuging blood collected from 10 healthy BALB/c mice $(0.1 \mathrm{~mL}$ per mouse). siRNA nanocomplexes suspended in PBS at a ratio of 1:200 were incubated with $\mathrm{RBCs}$ at $37^{\circ} \mathrm{C}$ for $3 \mathrm{~h}$. The absorbance $(\mathrm{X})$ of the supernatant from centrifuged samples was measured to estimate the amount of hemoglobin released from RBCs. RBCs were also treated with $1 \%$ Triton and PBS to provide positive and negative control samples, respectively. ${ }^{22}$ The formula for calculating the degree of hemolysis is as follows:

(X from complexes-X from $\mathrm{PBS}) /(\mathrm{X}$ from Triton-X from PBS) $\times 100 \%$.

\section{In vivo Cytocompatibility Assay}

$\mathrm{BALB} / \mathrm{c}$ mice (three per group) were injected with $100 \mu \mathrm{L}$ $\mathrm{Ca}^{2+}$-siRNA ( $5 \mathrm{mM} \mathrm{CaCl}_{2}, 100 \mathrm{nM}$ siRNA) alone or complexed with BSA $(0.025-25 \mu \mathrm{g} / \mathrm{mL})$ in the hind leg muscles.
In addition, Milli-Q water was used as a negative control. For histological evaluation, legs were collected $48 \mathrm{~h}$ after injection and fixed in 4\% paraformaldehyde. The sections were stained with hematoxylin and eosin (HE staining).

\section{Osteogenesis-Related Gene Expression}

Total RNA was extracted with TRIzol and complementary DNA (cDNA) was obtained using a reverse transcription kit (Qiagen, Germany), according to the manufacturer's instructions. OneStep RT-PCR Kit (Qiagen, Germany) and ABI 7500 system (Applied Biosystems, USA) were used for amplification and detection. Expression was determined by normalizing with the glyceraldehyde phosphate dehydrogenase $(G A P D H)$ gene, which acted as a control gene (encoding). The related primer sequences are listed in Table 2.

\section{Osteogenic Differentiation}

BSA-Ca ${ }^{2+}$-siWWP1, BSA-Ca ${ }^{2+}$-siNC, Lipo-siWWP1, and Milli-Q water were prepared. Three days after PDLSCs inoculation and transfection, osteogenic differentiation was performed in osteogenic medium (Biological Industries, Israel) with or without TNF- $\alpha(15 \mathrm{ng} / \mathrm{mL})$. After 2 weeks of induction, the medium was removed, and PDLSCs were fixed in 4\% paraformaldehyde for $20 \mathrm{~min}$. Then, the cells were stained with alizarin red S solution for $10 \mathrm{~min}$ at $25^{\circ} \mathrm{C}$. The sample was imaged through a stereo microscope (Olympus, Japan). Subsequently, the stain was dissolved with a cetylpyridinium chloride solution $(10 \mathrm{mM})$, and the absorbance at $580 \mathrm{~nm}$ was measured with a microplate reader.

\section{Statistical Analysis}

At least three independent experiments were performed per test, and statistical evaluations of data were performed using Student's $t$-test. All results were presented as mean \pm standard deviation, and $\mathrm{p}<0.05$ was considered statistically significant.

Table 2 Primers Used for Real-Time Quantitative Polymerase Chain Reaction

\begin{tabular}{|l|l|l|}
\hline Gene & Forward Primer Sequence (5'-3') & Reverse Primer Sequence (5'-3') \\
\hline WWPI & AGGCAGAGATCTGCTTGGTTGA & ACGCAGTCCTTTTTGAAGGCCA \\
ITCH & GCAGAGAGTGGACCAGCACG & TCCCATGTTGTCAACCCGCC \\
SmurfI & ACATCGTGCGGTGGTTCTGG & GCCGCGCCTGTAGAACCTT \\
Smurf2 & CTCAGGGGTGCCTCAGGAGT & GGGGTGGGGATATGGAGCCT \\
GAPDH & TGCACCACCAACTGCTTGC & GGCATGGACTGTGGTCATGAG \\
\hline
\end{tabular}

Abbreviations: WWPI, WW domain-containing E3 ubiquitin protein ligase I; ITCH, itchy E3 ubiquitin protein ligase; SmurfI, SMAD-specific E3 ubiquitin protein ligase I; Smurf2, SMAD-specific E3 ubiquitin protein ligase 2. 


\section{Results and Discussion}

\section{Synthesis and Characterization of} $\mathrm{BSA}-\mathrm{Ca}^{2+}$-siRNA Nanoparticles

$\mathrm{Ca}^{2+}$ may act as a bridge, connecting the negatively charged siRNA with the protein. SEM observation revealed that BSA, $\mathrm{Ca}^{2+}$, and siRNA formed spherical particles with an average diameter of $139.4 \pm 23.9 \mathrm{~nm}$ (Figure $1 \mathrm{~A}$ ). $\mathrm{Ca}^{2+}$ is combined with BSA or siRNA to form complexes with diameters of $156.6 \pm 28.5 \mathrm{~nm}$ (Figure 1B) and 112.1 $\pm 15.6 \mathrm{~nm}$ (Figure $1 \mathrm{C})$, respectively. AFM showed that the surface morphology of BSA-Ca ${ }^{2+}$-siRNA was rough, unlike that of $\mathrm{BSA}-\mathrm{Ca}^{2+}$ and $\mathrm{Ca}^{2+}$-siRNA (smooth surfaces). The formation of BSA$\mathrm{Ca}^{2+}$-siRNA nanoparticles was also observed by AFM. Notably, the surface morphology of BSA-Ca ${ }^{2+}$-siRNAs (Figure 1D) was irregular, and the particle sizes were intermediate, between those of $\mathrm{BSA}-\mathrm{Ca}^{2+}$ (Figure 1E) and $\mathrm{Ca}^{2+}$-siRNA (Figure 1F). This may be due to the disordered binding of BSA and $\mathrm{Ca}^{2+}$-siRNA. ${ }^{23}$

BSA, $\mathrm{Ca}^{2+}$, and siRNA can form nanocomplexes with a surface charge of $4.72 \pm 0.37 \mathrm{mV}$ in $\alpha$-MEM, as observed by DLS (Figure S1). Particles' diameter was similar to that determined by SEM. The results of FTIR are given in Figure 2. The infrared active vibration of biomolecules is as follows: 3,200-3,650 $\mathrm{cm}^{-1}$ asymmetric and symmetric O-H stretching; 3,250-3,300 $\mathrm{cm}^{-1}$ amide I (N-H stretch); 2,890-2,960 $\mathrm{cm}^{-1} \quad \mathrm{C}-\mathrm{H} \quad$ stretching of alkenes; $2,100-2,360 \mathrm{~cm}^{-1}$ triple bond (C-C or C-N) or cumulative double bond (C-C-C) stretch; 1,600-1,680 $\mathrm{cm}^{-1} \mathrm{C}-\mathrm{C}$ stretch (weak peaks) and $\mathrm{C}-\mathrm{O}$ amide stretch (sharp peaks); 1,480-1,590 $\mathrm{cm}^{-1}$ amide II $(\mathrm{N}-\mathrm{H}$ bend in plane or C-N stretch) $1,455 \mathrm{~cm}^{-1} \mathrm{C}-\mathrm{H}$ stretching of alkenes; $1,230-1,330 \mathrm{~cm}^{-1}$ amide III $(\mathrm{N}-\mathrm{H}$ bend in plane or C-N stretch); and 1,000-1,350 $\mathrm{cm}^{-1}$ amine C-N stretch. The structure of BSA-Ca ${ }^{2+}$-siRNA contained the maximum numbers of C-C or C-N stretching peaks, and several additional peaks, which were also at a maximum, including $3,430,2,358$, and $2,155 \mathrm{~cm}^{-1}$. However, the structure of BSA-Ca ${ }^{2+}$-siRNA did not contain peaks specific to BSAsiRNA, such as $1,539,1,455$, and $1,239 \mathrm{~cm}^{-1}$. This suggests that $\mathrm{BSA}, \mathrm{Ca}^{2+}$, and siRNA may form a complex rather than exist alone in the form of BSA-siRNA and $\mathrm{Ca}^{2+}$-siRNA (Figure 2).

\section{Loading and Release of siRNA}

Following BSA and $\mathrm{Ca}^{2+}$ nanoparticle formation, we evaluated the ability of BSA-Ca ${ }^{2+}$ to directly load siRNA. When the loading concentration of BSA was $\geq 500 \mu \mathrm{g} / \mathrm{mL}$, unbound
siRNA was only weakly detected in the gel (Figure 3A and B). Similarly, when BSA $(500 \mu \mathrm{g} / \mathrm{mL})$ and $\mathrm{CaCl}_{2}(1 \mathrm{M})$ were loaded with $100 \mathrm{nM}$ siRNA, unbound siRNA remained weakly detected in the gel, indicating that $\mathrm{BSA}-\mathrm{Ca}^{2+}$ can load a large amount of siRNA (Figure $3 \mathrm{C}$ and $\mathrm{D}$ ). In addition, a gel blocking test with various concentrations of $\mathrm{Ca}^{2+}(0.5-2$ M) loaded with siRNA (100 nM) revealed no clear bands (Figure $\mathrm{S} 2$ ). This suggested that the $\mathrm{Ca}^{2+}$-siRNA nanoparticles have poor stability. Conversely, the $\mathrm{BSA}-\mathrm{Ca}^{2+}$-siRNA nanoparticles could firmly retain and protect the siRNA cargo, thus confirming the stability of $\mathrm{BSA}-\mathrm{Ca}^{2+}$-loaded SiRNA.

We monitored the time/pH-related degradation of BSA$\mathrm{Ca}^{2+}$-siRNA using a spectrophotometer and found that BSA-Ca ${ }^{2+}$-siRNA particles could continuously unload siRNA, with release of $48 \%$ in neutral PBS by day 7 and $64 \%$ in acidic PBS (Figure $3 \mathrm{E}$ ). This suggests that exposure of BSA-Ca ${ }^{2+}$-siRNA particles to acidic subcellular compartments (such as endosomes/lysosomes) will enhance their degradability. This phenomenon may be attributed to the $\mathrm{pH}$ sensitivity of BSA. ${ }^{24}$ Under physiological conditions, BSA is adsorbed on the cation core by the negative charge on its surface. As the $\mathrm{pH}$ decreases, the electrostatic attraction between the inner core and the BSA gradually weakens. $^{25,26}$ Therefore, the $\mathrm{pH}$-responsive behavior of $\mathrm{BSA}-\mathrm{Ca}^{2+}$ promotes the intracellular release of siRNA.

\section{Cellular Uptake of Nanoparticles}

After confirming the effectiveness of siRNA loading/release behavior of BSA-Ca ${ }^{2+}$, in vitro experiments were conducted to evaluate the intracellular delivery of the loaded siRNA and to confirm the downregulation of target genes. To confirm the efficiency of BSA-Ca ${ }^{2+}$-siRNA silencing, the GFP gene was selected. As shown in Figure 4A, both Cy3 and FITC fluorescence were observed in cells of the experimental group, while only FITC fluorescence was observed in cells of the control group. This indicated that free siRNA cannot pass into the cell without $\mathrm{Ca}^{2+}$. However, $\mathrm{Cy} 3$-siGFP loaded with $\mathrm{BSA}-\mathrm{Ca}^{2+}$ can cause cells to emit a bright fluorescent signal, confirming that BSA-Ca ${ }^{2+}$ can successfully deliver siRNA in cells. Based on flow cytometry analysis, fluorescence could be observed in cells following the uptake of Cy3-siGFP. Compared with untransfected cells, the fluorescence signal of cells treated with BSA-Ca ${ }^{2+}$-siGFP increased significantly (Figure 4B and C).

To delineate the endocytosis pathway involved in the cellular uptake of BSA-Ca ${ }^{2+}$-siRNA, three specific inhibitors of endocytosis including chlorpromazine (CPZ), 

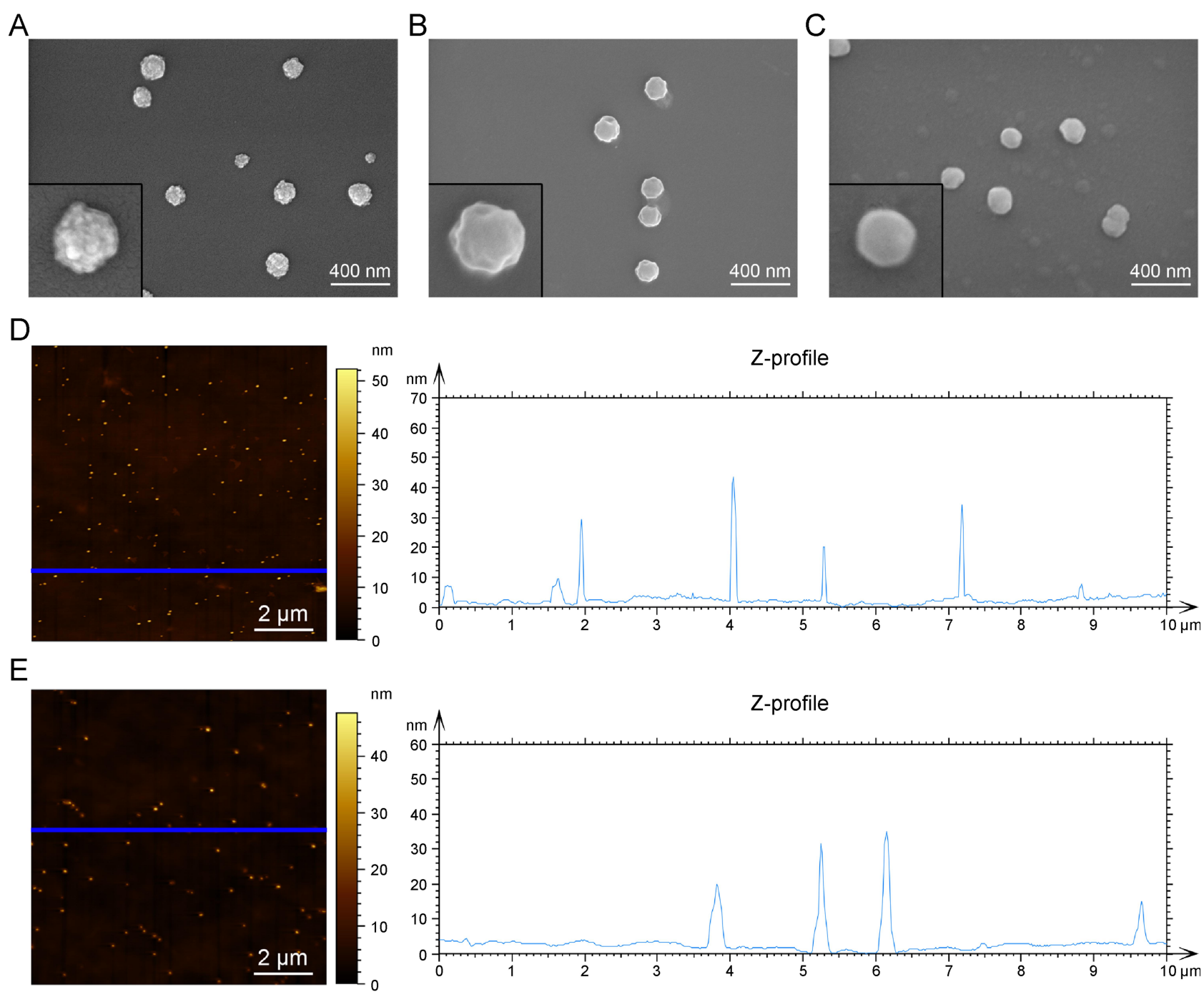

$\mathrm{F}$
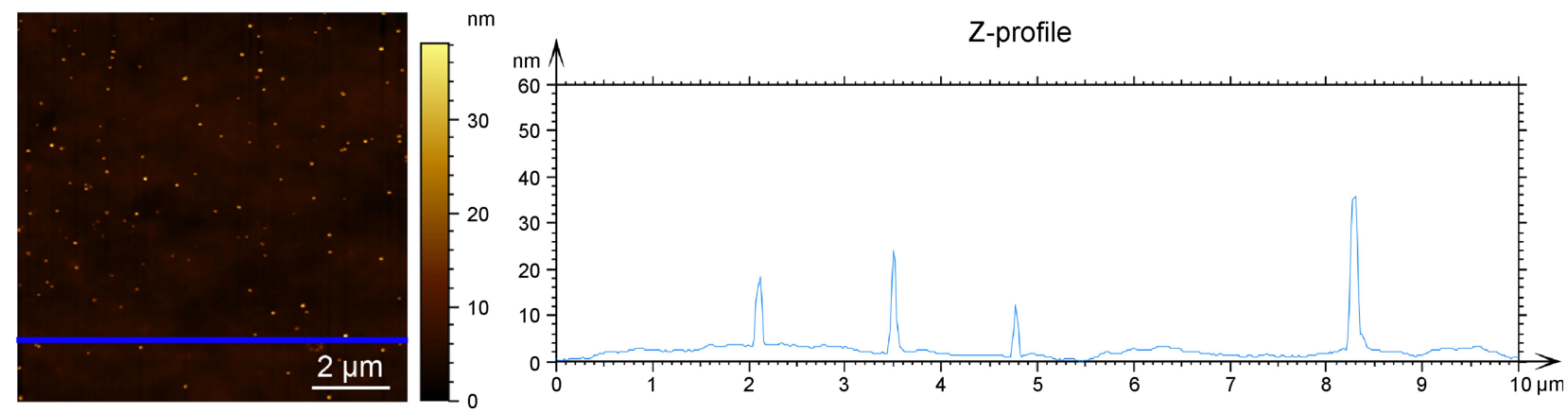

Figure I Scanning electron microscope (SEM) images of BSA-Ca ${ }^{2+}$-siRNA (A), BSA-Ca ${ }^{2+}$ (B), $\mathrm{Ca}^{2+}$-siRNA (C). Atomic force microscopy (AFM) images and Z-profile (D-F) of BSA-Caa ${ }^{2+}$-siRNA (D), BSA-Ca ${ }^{2+}(\mathbf{E}), \mathrm{Ca}^{2+}$-siRNA (F). Scale bars $=400 \mathrm{~nm}$ and $2 \mu \mathrm{m}$ (Blue line: a plane scanned in the Z-profile measurement).

genistein (GEN), and amiloride were used during the transfection process. The intracellular uptake of siRNA was further examined by flow cytometry. Quantitative analysis confirmed that cells pretreated with any inhibitor presented lower levels of siRNA uptake than untreated cells (Figure 4B). The inhibitory effect of amiloride on
siRNA uptake was the most notable and exceeded $70 \%$ (Figure 4C). This indicated that macropinocytosis is the main uptake mechanism. A recent study showed that consecutive macropinocytosis in macrophages is mediated by calcium-sensing receptors (CaSR). ${ }^{27}$ Despite the use of different cell types in that study, we speculate that the 


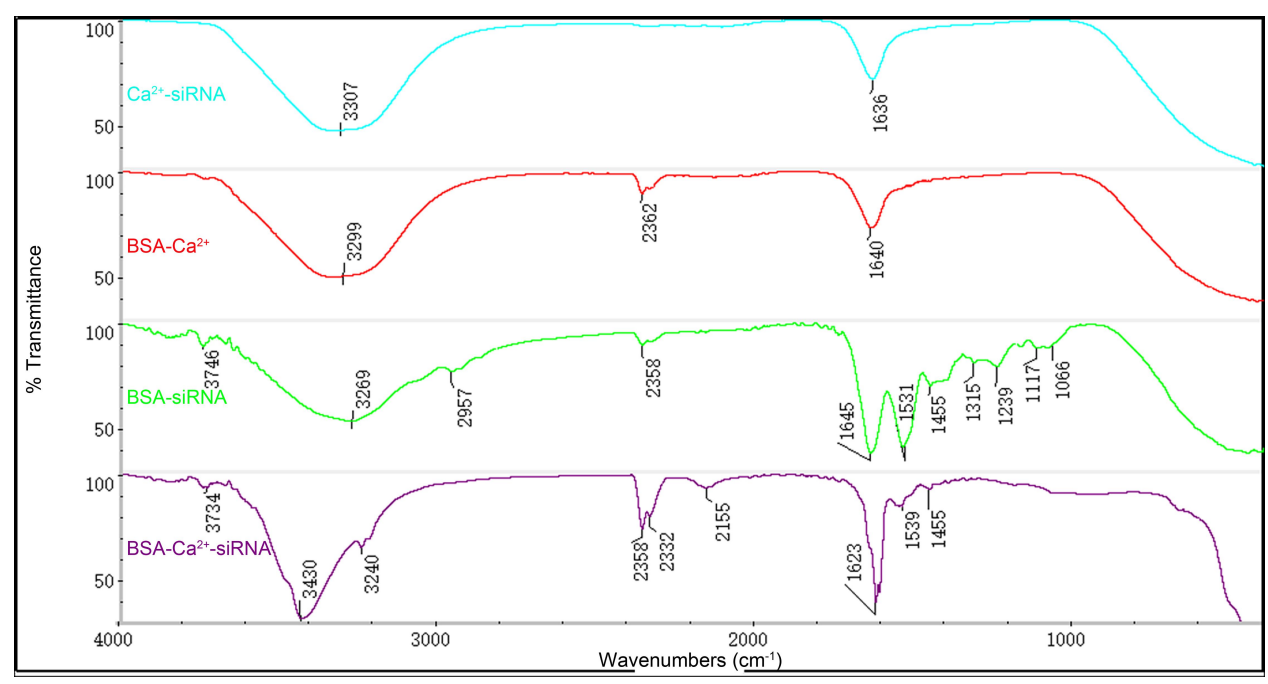

Figure 2 Fourier transform infrared spectroscopy (FTIR) spectra analyses of complexes.

endocytosis of BSA-Ca ${ }^{2+}$-siRNA may involve the activation of CaSR. However, further studies are needed to elucidate the exact mechanism underlying the endocytosis of $\mathrm{BSA}_{-} \mathrm{Ca}^{2+}{ }^{2+}$ siRNA and subsequent release from endosomes.

\section{Intracellular Release of Nanoparticles}

The intracellular release of $\mathrm{Cy} 3$-siRNA was evaluated by LSCM. Following the uptake of BSA-Ca ${ }^{2+}$-siRNA by cells, a large amount of siRNA was found to be internalized and localized on endosomes/lysosomes (Figure 5A). This indicated that the endosome/lysosome is involved in the release of siRNA. To clarify the escape mechanism of nanoparticles from endosomes, two inhibitors were used: bafilomycin A1, which is a V-ATPase inhibitor that prevents endosome acidification, and YM201636, a PIKfyve inhibitor that prevents early endosomes from maturing into late endosomes. Using fluorescence microscopy, bafilomycin A1 treatment was found to inhibit the silencing effect of siGFP on GFP in PDLSCs, unlike treatment without inhibitor (Figure 5B). The release of siRNA in cells was further evaluated by flow cytometry. Quantitative analysis confirmed that bafilomycin A1 caused a more significant reduction in GFP silencing than YM201636 following 24 $\mathrm{h}$ of treatment (Figure 5C and D). This indicated that siRNA release may be primarily mediated by the acidification of endosomes/lysosomes promoting the decomposition of nanocomplexes. After uncomplexing, free $\mathrm{Ca}^{2+}$ increases rapidly and promotes the entry of protons into the endosome/lysosome, causing the rupture of the endosome and the release of siRNA into the cytoplasm. This process of protons entering the endosome/lysosome to replace $\mathrm{Ca}^{2+}$ is termed the "proton sponge" effect. ${ }^{28,29}$ The rapid de-complexation and siRNA release in the early endosomes are beneficial for maintaining siRNA activity and the high efficiency of gene silencing induced by BSA-Ca ${ }^{2+}$-siRNA nanocomplexes.

\section{Evaluation of the Cytocompatibility of Complexes}

In previous transfection experiments, high $\mathrm{Ca}^{2+}$ concentrations and FBS-free medium were found to induce cell death (Figure S3). Therefore, we optimized $\mathrm{Ca}^{2+}$-siRNA with BSA coating to evaluate the effect of BSA in reducing the cytotoxicity of high $\mathrm{Ca}^{2+}$ concentrations. We found that the carrier with high concentration of BSA $(\geq 500 \mu \mathrm{g} / \mathrm{mL})$ did not significantly affect cell viability. In contrast, the nanoparticles with low BSA concentration $(\leq 50 \mu \mathrm{g} / \mathrm{mL})$ were found to have high cytotoxicity (Figure 6A). This indicated that BSA can improve the cellular compatibility of $\mathrm{Ca}^{2+}$-siRNA.

The working solution was diluted with PBS at a ratio of 1:200 to obtain final concentrations for cell hemolysis experiments. No significant hemoglobin leakage was detected in RBCs incubated with the $\mathrm{Ca}^{2+}$-siRNA containing $5,000 \mu \mathrm{g} / \mathrm{mL}$ BSA. In contrast, dose-dependent hemoglobin leakage was observed in RBCs incubated with transfection solution with the BSA concentration of $\leq 500$ $\mu \mathrm{g} / \mathrm{mL}$ (Figure 6B). Furthermore, HE staining revealed that decreasing the BSA concentration in the transfection solution resulted in a dose-dependent inflammatory response and necrosis in muscle tissue (Figure 6C). This indicated that BSA can improve the biocompatibility of 
A

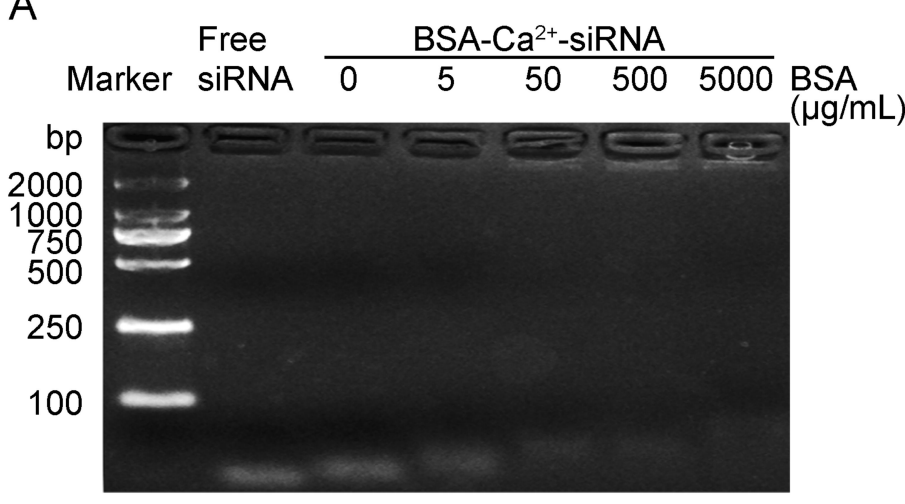

C

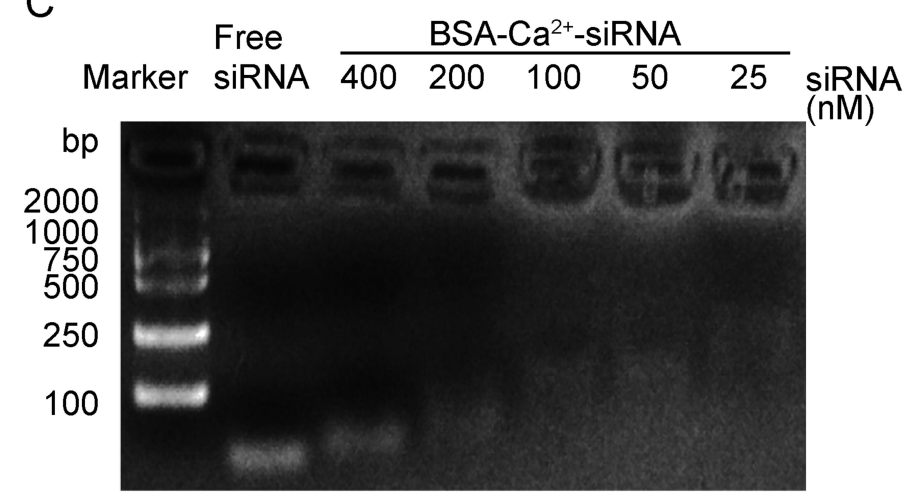

E

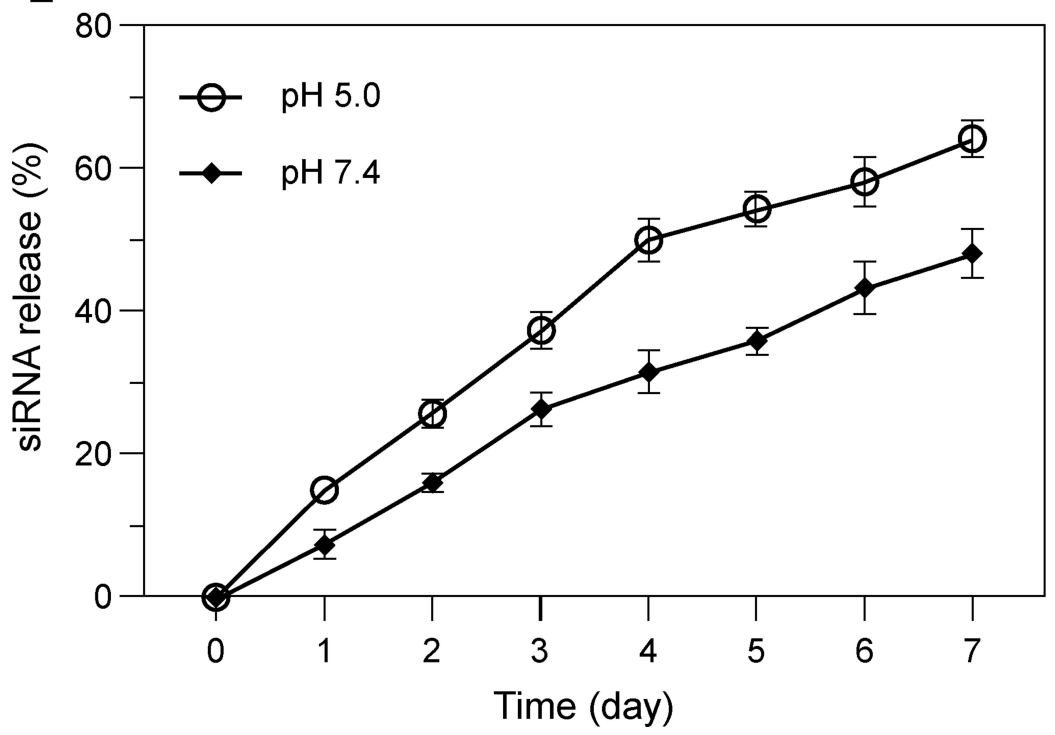

B

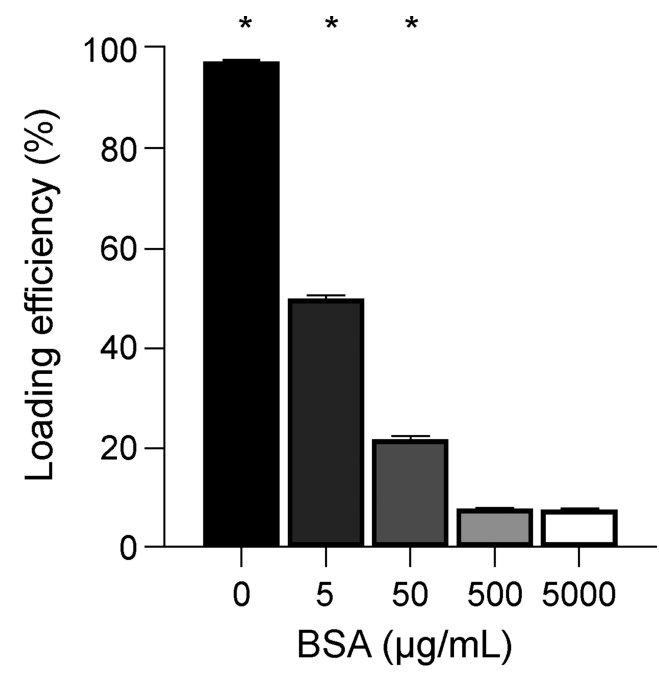

D

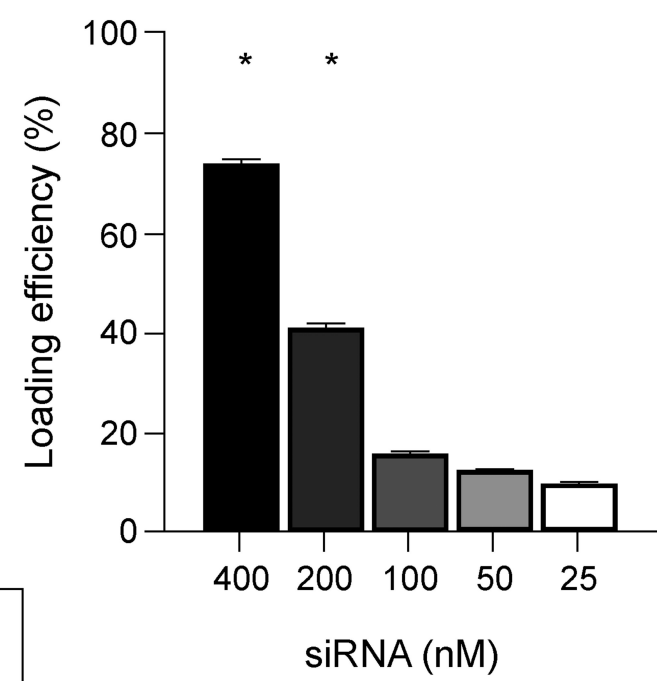

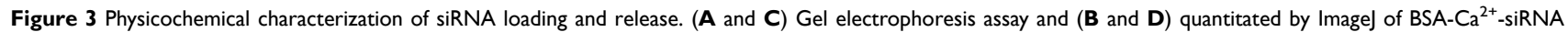
processed at various concentrations of bovine serum albumin (BSA) $(0-5000 \mu \mathrm{g} / \mathrm{mL})$ or siRNA (25-400 $\mathrm{nM}$ ). (E) Release profiles of siRNA from BSA-Ca ${ }^{2+}$ in PBS (I mg/mL) at different $\mathrm{pH}$. Significance: $* \mathrm{p}<0.05$.

$\mathrm{Ca}^{2+}$-siRNA and suggested that BSA-Ca ${ }^{2+}$ may represent a siRNA delivery platform with favorable biological safety. Thus, this carrier may provide a feasible solution for use in the hypodermic, intramuscular, and intravenous injection of siRNA drugs for the purpose of gene therapy. ${ }^{30,31}$ 

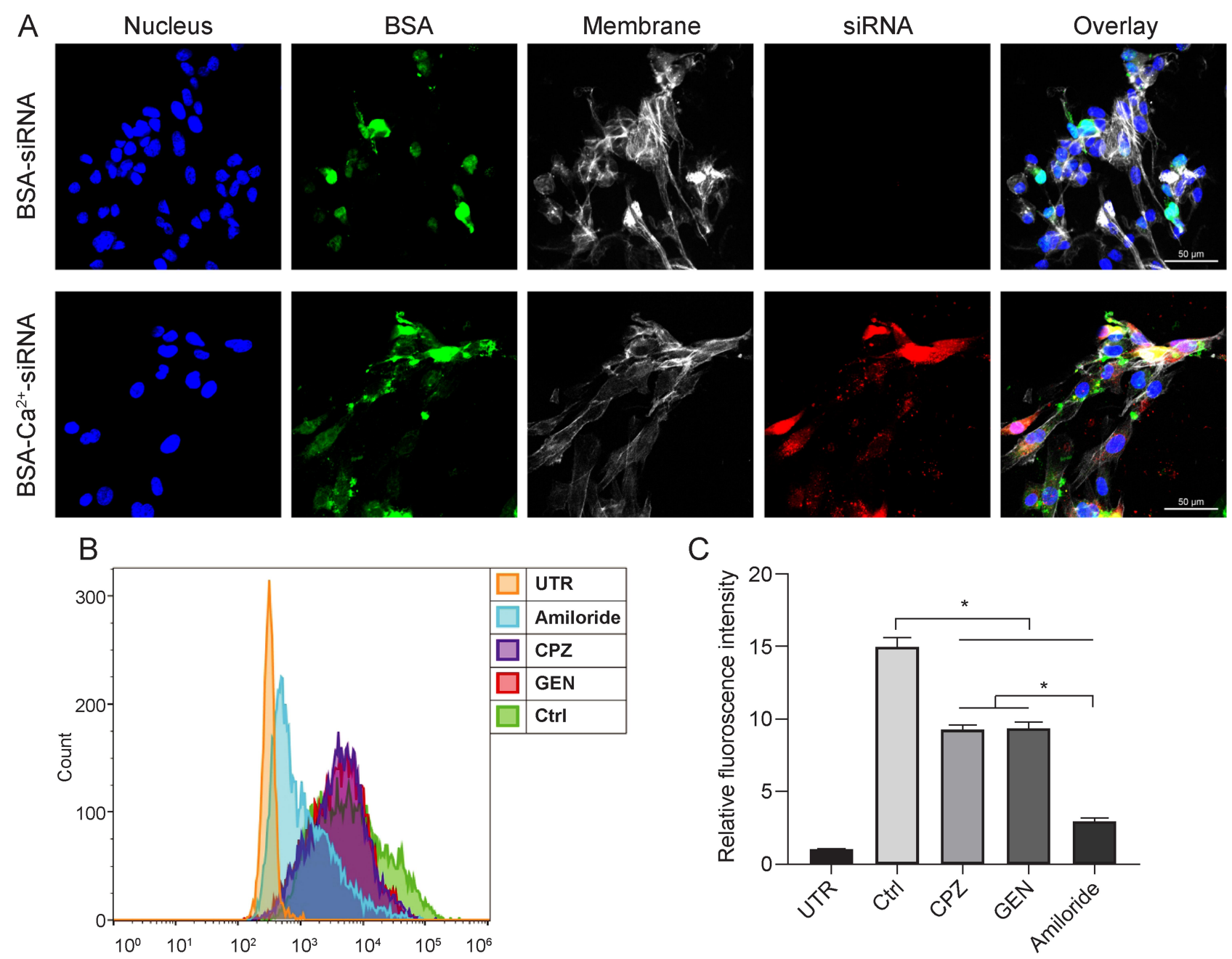

Figure 4 Mechanisms of intracellular uptake and endocytosis of FITC-BSA-Ca ${ }^{2+} / \mathrm{Cy} 3$-siRNA complexes in periodontal ligament stem cells (PDLSCs). (A) PDLSCs were stained with AF635-phalloidin and Hoechst and examined under laser scanning confocal microscope (LSCM). (B and C) PDLSCs were pretreated with inhibitors for I h and then incubated with transfection complexes for another $3.5 \mathrm{~h}$. Transfection without inhibitors was used as a control (Ctrl). Cellular uptake was analyzed by flow cytometry analysis (B) and quantitated (C). Geometric mean of fluorescence intensity was normalized by untransfected cells (UTR). Significance: *P < 0.05 .

Thus, the fabrication scheme shown in Figure 7 describes our proposed vision of BSA-Ca ${ }^{2+}$-siRNA nanocomplexes, which comprise a BSA coating on the surface of $\mathrm{Ca}^{2+}$-siRNA nanoparticles. BSA is essential for reducing the toxicity associated with high $\mathrm{Ca}^{2+}$ concentrations and drives the uptake of nanoparticles by cells. The mechanism underlying the internalization of nanoparticles involves a variety of endocytic pathways including clathrin-mediated endocytosis, caveolae-mediated endocytosis, and macropinocytosis. Subsequently, the siRNA released from the endosome reaches the cytosol where the RNA-induced silencing complex is located, resulting in effective siRNA-induced silencing of the target gene. In conclusion, BSA-Ca ${ }^{2+}$-siRNA transfection is a convenient, safe, and effective method for delivering siRNA/miRNA to PDLSCs.

\section{Inducing Osteogenic Differentiation of Inflammatory PDLSCs}

Having established the gene silencing effect through cytosolic siRNA delivery using endosome/lysosome-disruptive BSA-Ca ${ }^{2+}$ carriers, we further evaluated the method in vitro using a PDLSC osteogenic differentiation assay. Inflammatory cytokines, such as TNF- $\alpha$, impair the osteogenic differentiation ability of MSCs by mediating the upregulation of the WWP1 gene ${ }^{17}$ and downregulation of the ALP, COL-1, and OCN genes. ${ }^{32}$ This indicates that the ubiquitin-proteasome pathway may negatively influence osteoblast differentiation. Using real-time quantitative polymerase chain reaction ( $\mathrm{qPCR}$ ), we found that the mRNA expression of WWP1, Smurf1, and Smurf2 in PDLSCs was significantly up-regulated 7 days post 

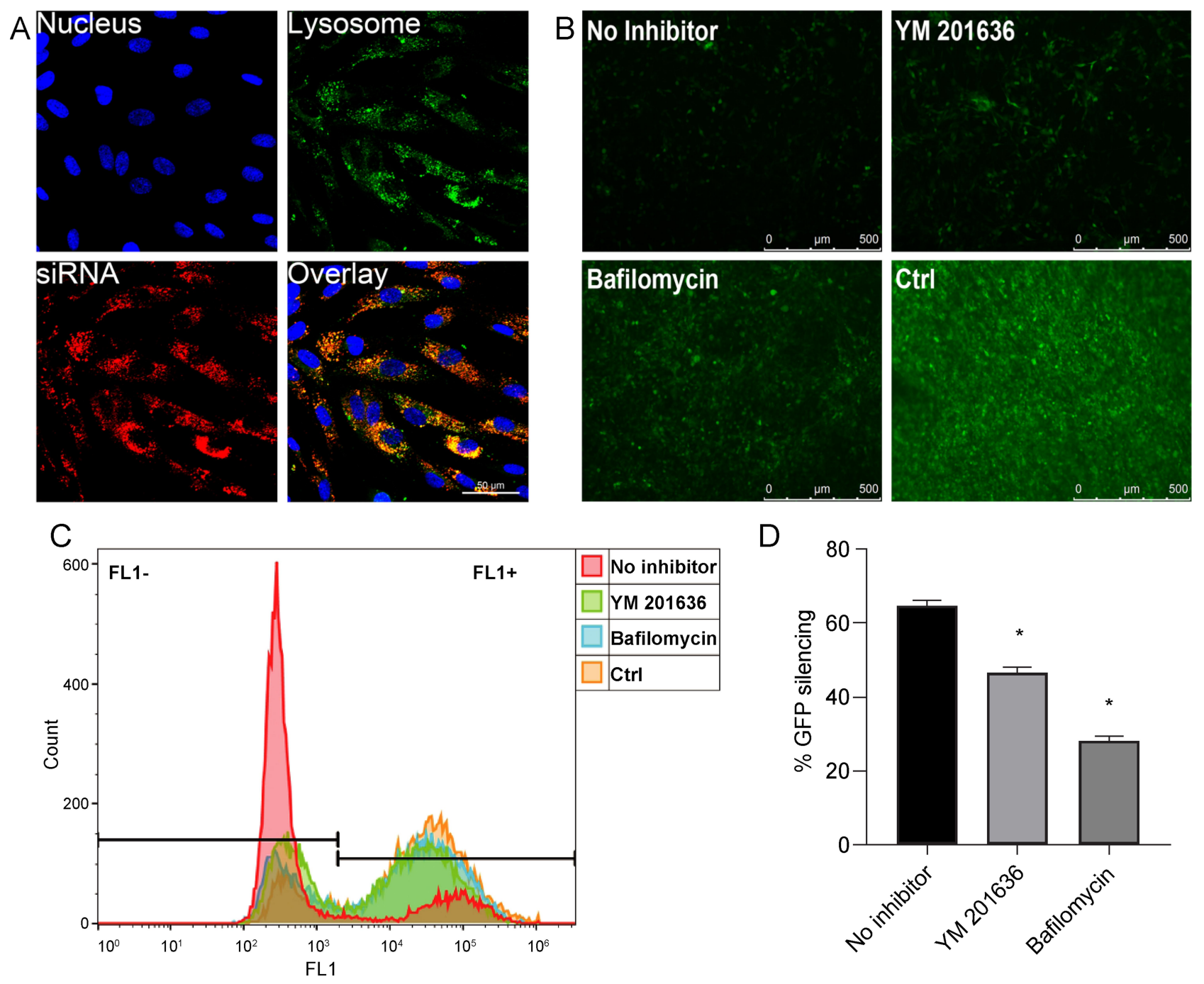

Figure 5 (A) Fluorescence images of cells treated with BSA-Ca ${ }^{2+}$-siRNA (Blue: Hoechst-stained nuclei; Green: LysoTracker-stained endosomes/lysosomes; Red: Cy3siRNA). GFP silencing at $24 \mathrm{~h}$ post-transfection in GFP-PDLSCs and the effect of inhibitors on endosomal release were analyzed by fluorescence microscopy (B) and flow cytometry (C) and quantitated (D). The silencing effect was normalized by GFP-PDLSCs with siNC (Ctrl). Significance: *P < 0.05 .

treatment with TNF- $\alpha$. Notably, on day 14, WWP1 gene expression remained increased (Figure 8A). This suggested that WWP1 may have a large effect on the inflammatory response of PDLSCs induced by TNF- $\alpha$.

We utilized the novel technology of BSA-Ca ${ }^{2+}$-siWWP1 particle therapy to inhibit the effect of TNF- $\alpha$ and restore the osteogenic differentiation ability of PDLSCs by blocking the activity of WWP1. Alizarin red staining showed that BSA$\mathrm{Ca}^{2+}$-siWWP1 significantly promoted the osteogenic differentiation of inflammatory PDLSCs (Figure 8B); there was no significant difference in the transfection efficiency between BSA-Ca ${ }^{2+}$ and Lipofectamine 2000 (Figure 8C). These results indicated that the revised-BSA optimized $\mathrm{Ca}^{2+}$-siWWP1 nanoparticles have high transfection efficiency and may promote the mineralization of bone defects that occur in periodontitis. Notably, bone resorption inhibition and bone formation promotion are crucial for treating periodontal inflammation. Therefore, siWWP1 can prevent TNF- $\alpha$ from inhibiting bone formation and can enhance the osteogenic effect of PDLSCs; moreover, it may be beneficial for regenerating and repairing bone tissue defects accompanying periodontitis.

There are some limitations to this study. Although we observed the formation of $\mathrm{BSA}-\mathrm{Ca}^{2+}$-siRNA nanocomplexes, the binding strength among $\mathrm{BSA}, \mathrm{Ca}^{2+}$, and siRNA could not be determined. If such binding is stable, BSA can be modified using antibodies directed against the surface markers of target cells for targeted delivery. In addition, the transfection mechanism of formulated nanoparticles could not be fully elucidated. Moreover, the toxicity and 
A

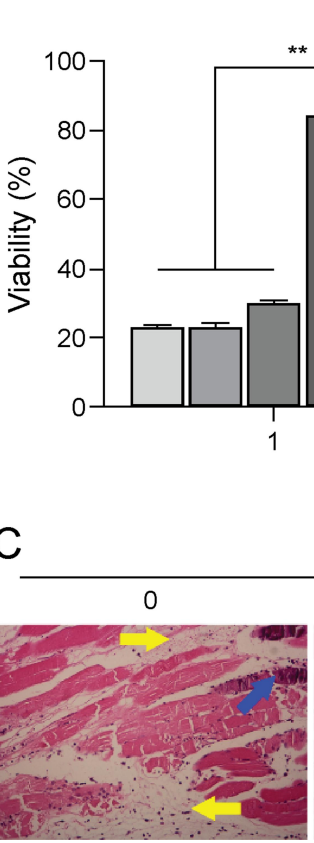

a-MEM

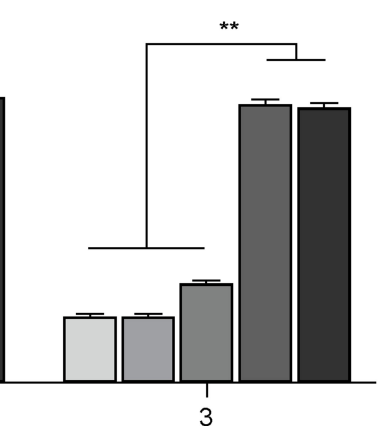

Time (day)
B

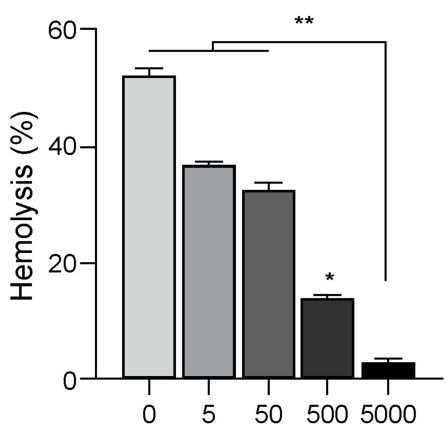

BSA Concentration $(\mu \mathrm{g} / \mathrm{mL})$

Figure 6 (A) Cytotoxicity of PDLSCs treated with particles composed of different BSA concentrations (0-5000 ug/mL) after days I and 3 was determined by the CCK-8 method. (B) After $6 \mathrm{~h}$ of incubation, the hemolysis of BSA-Ca ${ }^{2+}$ was measured using red blood cells (RBCs) obtained from BALB/c mice ( $2 \%$ hematocrit). The data presents the mean \pm SD of the results obtained from four samples. (C) Hematoxylin and eosin (HE) staining of mice muscle tissue (yellow arrows: necrotic muscle cells, blue arrows: infiltrating inflammatory cells). Significance: $* \mathrm{P}<0.05, * * \mathrm{P}<0.01$.

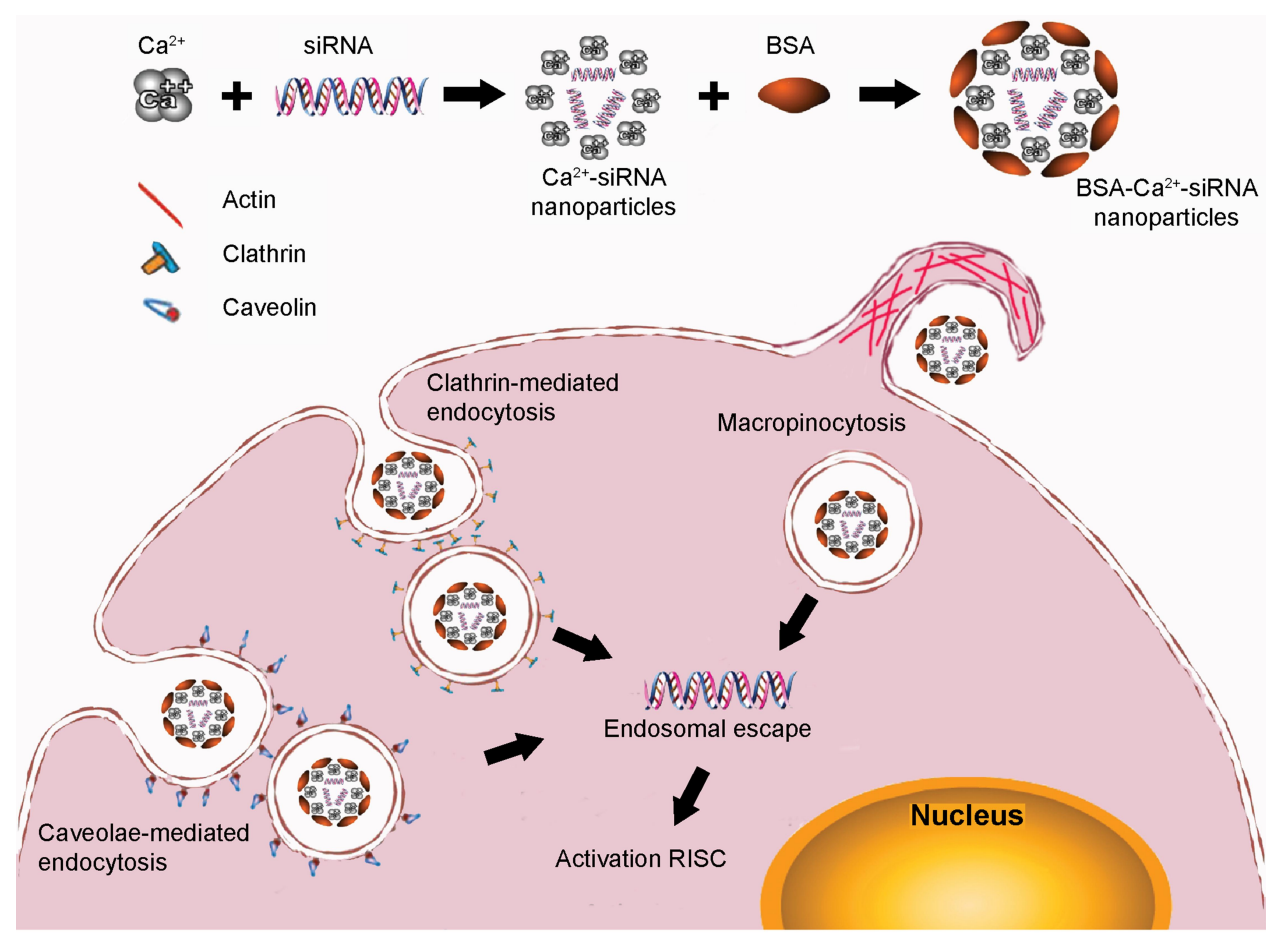

Figure 7 Proposed mechanism for BSA-Ca ${ }^{2+}$-siRNA nanocomplex assembly, cellular uptake, and release. RISC, RNA-induced silencing complex.

transfection efficiency of BSA-Ca ${ }^{2+}$-siRNA in other cells, such as tumor cells, are yet to be evaluated. Finally, the in vivo microenvironment is complex, containing numerous phosphates and proteins; therefore, whether these substances affect the stability, biocompatibility, and transfection efficiency of BSA-Ca ${ }^{2+}$-siRNA remains to be determined. 


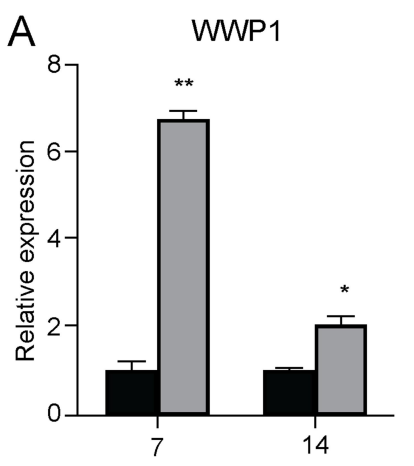

Differentiation days

Smurf1

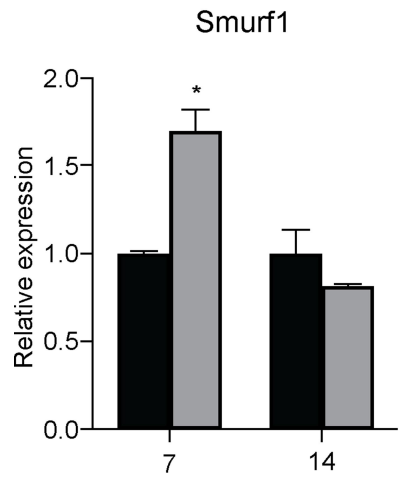

Differentiation days

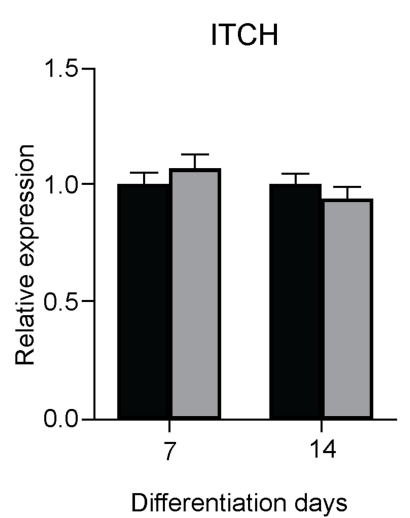

Smurf2

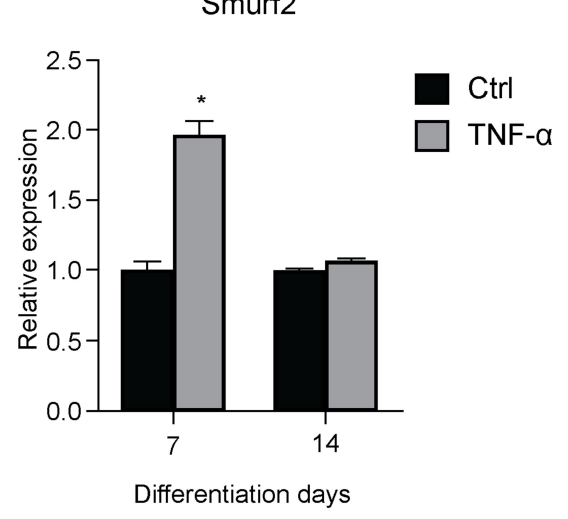

B
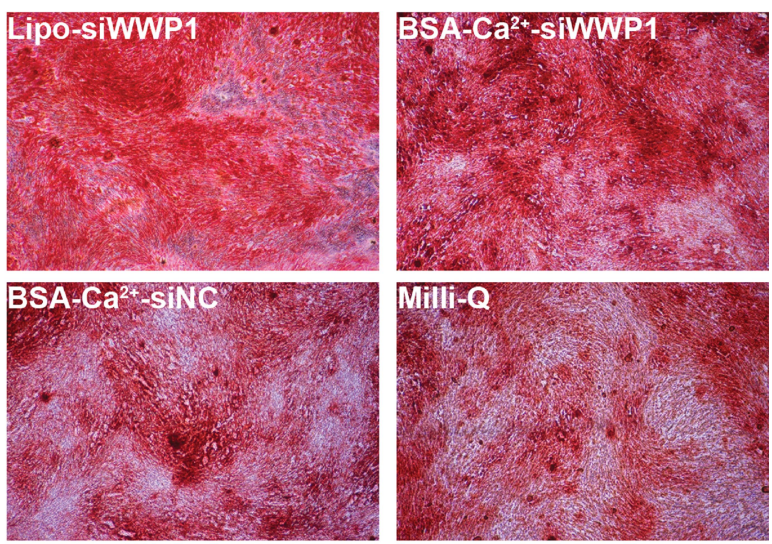

Mili-Q

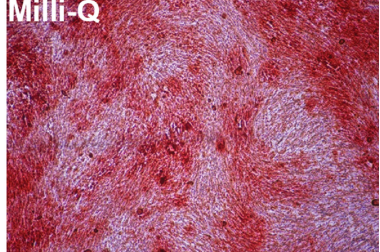

C

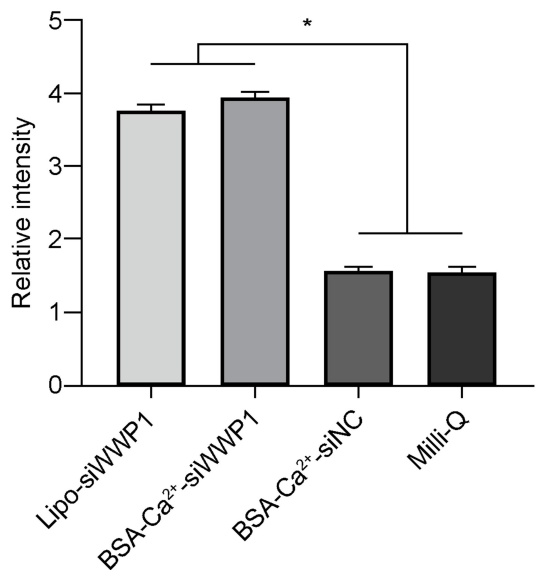

Figure 8 Increased expression of WWPI in PDLSCs stimulated by tumor necrosis factor (TNF)- $\alpha$. (A) The expression of osteogenesis-related genes (WWPI, ITCH, SmurfI, and Smurf2) was determined by qPCR on days 7 and I4. The expression level of mRNA was normalized to that of GAPDH. Triplicate values are shown, and the data are presented as mean \pm SD. PDLSCs were stimulated with TNF- $\alpha$ and transfected with WWPI siRNA or NC siRNA, and cultured in osteoblast differentiation medium for 2 weeks. (B) Analysis of osteogenic differentiation in vitro by Alizarin Red staining. (C) Evaluation of target gene expression by qPCR. Values are the mean \pm SD from three wells. The experiments were repeated three times with similar results. Significance: $* \mathrm{P}<0.05, * * \mathrm{P}<0.0 \mathrm{I}$.

\section{Conclusion}

BSA incorporation does not affect $\mathrm{Ca}^{2+}$-siRNA uptake or knockout efficiency and may reduce the adverse effects associated with high concentrations of $\mathrm{Ca}^{2+}$ in vivo. Therefore, we developed a convenient, safe, and effective siRNA delivery system which can effectively encapsulate and protect siRNA from degradation. The $\mathrm{pH}$-responsive behavior of BSA-Ca ${ }^{2+}$-siRNA promotes effective siRNA release into the cytoplasm, leading to durable and efficient gene silencing. Therefore, BSA-Ca ${ }^{2+}$ nanoparticles might be used as a platform to deliver siRNA into the cytoplasm for multiple gene targets and as a nanomaterial with high biocompatibility for intravenously injected drugs.

\section{Funding}

The authors are grateful for grants from the School of Stomatology, Air Force Medical University. This work was also supported by a grant from the Nature Science Foundation of China (NSFC No. 81771069) and the
Fundamental Research Funds for the Central Universities (xzy012019097).

\section{Disclosure}

The authors report no conflicts of interest in this work.

\section{References}

1. Yalcin E, Kara G, Celik E, et al. Preparation and characterization of novel albumin-sericin nanoparticles as siRNA delivery vehicle for laryngeal cancer treatment. Prep Biochem Biotechnol. 2019;49:659-670. doi:10.1080/10826068.2019.1599395

2. Ruvinov E, Kryukov O, Forti E, Korin E, Goldstein M, Cohen S. CalciumsiRNA nanocomplexes: what reversibility is all about. J Control Release. 2015;203:150-160. doi:10.1016/j.jconrel.2015.02.029

3. Putnam D. Design and development of effective siRNA delivery vehicles. Proc Natl Acad Sci U S A. 2014;111:3903-3904. doi:10.1073/pnas.1401746111

4. Xue HY, Liu S, Wong HL. Nanotoxicity: a key obstacle to clinical translation of siRNA-based nanomedicine. Nanomedicine (Lond). 2014;9:295-312.

5. Goldshtein M, Forti E, Ruvinov E, Cohen S. Mechanisms of cellular uptake and endosomal escape of calcium-siRNA nanocomplexes. Int J Pharm. 2016;515:46-56. doi:10.1016/j.ijpharm.2016.10.009 
6. Xu B, Xu Y, Su G, Zhu H, Zong L. A multifunctional nanoparticle constructed with a detachable albumin outer shell and a redox-sensitive inner core for efficient siRNA delivery to hepatocellular carcinoma cells. J Drug Target. 2018;26:941-954. doi:10.1080/ 1061186X.2018.1455840

7. Song W, Yang C, Svend Le DQ, Zhang Y, Kjems J. CalciummicroRNA complex-functionalized nanotubular implant surface for highly efficient transfection and enhanced osteogenesis of mesenchymal stem cells. ACS Appl Mater Interfaces. 2018;10:7756-7764. doi:10.1021/acsami.7b18289

8. Mammadova-Bach E, Nagy M, Heemskerk JWM, Nieswandt B, Braun A. Store-operated calcium entry in thrombosis and thrombo-inflammation. Cell Calcium. 2019;77:39-48. doi:10.1016/j. ceca.2018.11.005

9. Dalal PJ, Muller WA, Sullivan DP. Endothelial cell calcium signaling during barrier function and inflammation. $\mathrm{Am} J$ Pathol. 2020;190:535-542. doi:10.1016/j.ajpath.2019.11.004

10. Elzoghby AO, Samy WM, Elgindy NA. Albumin-based nanoparticles as potential controlled release drug delivery systems. J Control Release. 2012;157:168-182.

11. Bahrami M, Morris MB, Day ML. Amino acid supplementation of a simple inorganic salt solution supports efficient in vitro maturation (IVM) of bovine oocytes. Sci Rep. 2019;9:11739. doi:10.1038/ s41598-019-48038-y

12. Tarhini M, Greige-Gerges H, Elaissari A. Protein-based nanoparticles: from preparation to encapsulation of active molecules. Int J Pharm. 2017;522:172-197. doi:10.1016/j.ijpharm.2017.01.067

13. Verma ML, Dhanya BS, Rani V, et al. Carbohydrate and protein based biopolymeric nanoparticles: current status and biotechnological applications. Int J Biol Macromol. 2020;154:390-412. doi:10.1016/j. ijbiomac.2020.03.105

14. Nair AB, Kaushik A, Attimarad M, Al-Dhubiab BE. Enhanced oral bioavailability of calcium using bovine serum albumin microspheres. Drug Deliv. 2012;19:277-285. doi:10.3109/10717544.2012.704094

15. Glimcher LH, Jones DC, Wein MN. Control of postnatal bone mass by the zinc finger adapter protein Schnurri-3. Ann N Y Acad Sci. 2007;1116:174-181. doi:10.1196/annals.1402.044

16. Zhi X, Chen C. WWP1: a versatile ubiquitin E3 ligase in signaling and diseases. Cell Mol Life Sci. 2012;69:1425-1434. doi:10.1007/ s00018-011-0871-7

17. Zhao L, Huang J, Zhang H, et al. Tumor necrosis factor inhibits mesenchymal stem cell differentiation into osteoblasts via the ubiquitin E3 ligase Wwp1. Stem Cells. 2011;29:1601-1610. doi: $10.1002 /$ stem. 703

18. Tucker WO, Kinghorn AB, Fraser LA, Cheung YW, Tanner JA. Selection and characterization of a DNA aptamer specifically targeting human HECT ubiquitin ligase WWP1. Int $J$ Mol Sci. 2018;19:763. doi:10.3390/ijms19030763

19. Shu L, Zhang H, Boyce BF, Xing XL. Ubiquitin E3 ligase Wwp1 negatively regulates osteoblast function by inhibiting osteoblast differentiation and migration. J Bone Miner Res. 2013;28:1925-1935. doi:10.1002/jbmr.1938
20. Choi E, Lim D-K, Kim S. Calcium-doped mesoporous silica nanoparticles as a lysosomolytic nanocarrier for amine-free loading and cytosolic delivery of siRNA. J Ind Eng Chem. 2020;81:71-80. doi:10.1016/j.jiec.2019.08.054

21. Yang C, Gao S, Dagnæs-Hansen F, Jakobsen M, Kjems J. Impact of PEG chain length on the physical properties and bioactivity of PEGylated chitosan/siRNA nanoparticles in vitro and in vivo. ACS Appl Mater Interfaces. 2017;9:12203-12216. doi:10.1021/acsami.6b16556

22. Ma B, Niu C, Zhou Y, et al. The disulfide bond of the peptide thanatin is dispensible for its antimicrobial activity in vivo and in vitro. Antimicrob Agents Chemother. 2016;60:4283-4289. doi:10.1128/AAC.00041-16

23. Qin PH, Zhang W, Lu WC. Theoretical study of hydrated $\mathrm{Ca} 2$ +-amino acids (glycine, threonine and phenylalanine) clusters. Comput Theor Chem. 2013;1021:164-170. doi:10.1016/j. comptc.2013.07.005

24. Anders CB, Chess JJ, Wingett DG, Punnoose A. Serum proteins enhance dispersion stability and influence the cytotoxicity and dosimetry of $\mathrm{ZnO}$ nanoparticles in suspension and adherent cancer cell models. Nanoscale Res Lett. 2015;10:448. doi:10.1186/s11671-015-1158-y

25. Xu Y, Jin X, Ping Q, et al. A novel lipoprotein-mimic nanocarrier composed of the modified protein and lipid for tumor cell targeting delivery. J Control Release. 2010;146:299-308. doi:10.1016/j. jconrel.2010.05.022

26. Peng Q, Zhang S, Yang Q, et al. Preformed albumin corona, a protective coating for nanoparticles based drug delivery system. Biomaterials. 2013;34(33):8521-8530. doi:10.1016/j. biomaterials.2013.07.102

27. Canton J, Schlam D, Breuer C, et al. Calcium-sensing receptors signal constitutive macropinocytosis and facilitate the uptake of NOD2 ligands in macrophages. Nat Commun. 2016;7(1):11284. doi: $10.1038 /$ ncomms 11284

28. Dominska M, Dykxhoorn DM. Breaking down the barriers: siRNA delivery and endosome escape. J Cell Sci. 2010;123:1183-1189. doi:10.1242/jcs.066399

29. Chou LY, Ming K, Chan WC. Strategies for the intracellular delivery of nanoparticles. Chem Soc Rev. 2011;40:233-245. doi:10.1039/ C0CS00003E

30. Han J, Wang Q, Zhang Z, Gong T, Sun X. Cationic bovine serum albumin based self-assembled nanoparticles as siRNA delivery vector for treating lung metastatic cancer. Small. 2014;10(3):524-535. doi:10.1002/smll.201301992

31. Xu K, Zhao Z, Zhang J, et al. Albumin-stabilized manganese-based nanocomposites with sensitive tumor microenvironment responsivity and their application for efficient SiRNA delivery in brain tumors J Mater Chem B. 2020;8(7):1507-1515. doi:10.1039/C9TB02341K

32. Zhang J, Li ZG, Si YM, Chen B, Meng J. The difference on the osteogenic differentiation between periodontal ligament stem cells and bone marrow mesenchymal stem cells under inflammatory microenvironments. Differentiation. 2014;88:97-105. doi:10.1016/j. diff.2014.10.001
International Journal of Nanomedicine

\section{Publish your work in this journal}

The International Journal of Nanomedicine is an international, peerreviewed journal focusing on the application of nanotechnology in diagnostics, therapeutics, and drug delivery systems throughout the biomedical field. This journal is indexed on PubMed Central, MedLine, CAS, SciSearch ${ }^{\mathbb{R}}$, Current Contents ${ }^{\mathbb{B}} /$ Clinical Medicine,
Journal Citation Reports/Science Edition, EMBase, Scopus and the Elsevier Bibliographic databases. The manuscript management system is completely online and includes a very quick and fair peer-review system, which is all easy to use. Visit http://www.dovepress.com/ testimonials.php to read real quotes from published authors. 